VIII.

\title{
Zur Radikaloperation des Ductus omphalo-entericus persistens.
}

\author{
Von \\ Dr. Max Sträter, \\ Chirurg in Amsterdam.
}

Von den verschiedenen congenitalen Erkrankungen des Nabels zehört das Offenbleiben des Ductus omphalo-entericus zu den ziemlich selten vorkommenden, so daß der einzelne Fall noch immer casuistisches Interesse bietet. Ich erlaube mir also im folgenden einen Fall mitzuteilen, den ich vor einiger Zeit als Assistent der chirurgischen Abteilung des 0. L. V. Gasthuis in Amsterdam zu beobachten und za operieren Gelegenheit hatte.

Die Krankengeschichte lautet wie folgt:

Patient wurde am 4. September 1902 geboren. Dic Geburt verlief spontan. Schon bald nach der Geburt fiel es der Mutter auf, daß etwas am Nabel des Kindes nicht in Ordnung war, und sie brachte es deswegen, als es 14 Tage alt war, zur Poliklinik des Krankenhauses. Es befand sich damals am Nabel des Kindes eine ungefähr haselnußgroße Geschwulst, die zum größten 'Teile noch mit nekrotischen Resten der Nabelschnur bedeckt. war. Fine Woche später hatte sich alles Necrotische abgestoßen, und der Status war jetzt der folgende: Patient ist ein kräftig entwickelter Knabe. Gewicht 9 Pfund. Merz und Lungen sind normal. Fr wird von der Mutter genährt. Seine Verdaung ist vollkommen in Ordnung.

Die Stelle des Nabels ist eingenommen von einem ungefähr haselnußgroßen Tumor, dessen Oberfläche makroskopiseh wie Darmschleimhaut aussieht. Die Consistenz des 'Tumors ist elastisch; eine Reposition des Tumors läßt sich nicht zustande bringen; er wird nicht deutlich größer bei Aetion der Bauehpresse. Etwas nach links von der Spitze des Tumors befindet sich eine Öffnung, worin eine feine Knopfsonde, otme den geringsten Widerstand zu finden, sich viele Zentimeter weit bauchwärts, und zwar nach rechts oben, einführen läßt. Aus dieser Öffnung tritt etwas klarer Schleim hervor. Darminhalt ist nach Angabe der Mutter nie heransgekommen. Links von diesem Tumor befindet sich ein erbsengroßes Granulom. Sonst ist auch am Abdomen des Kindes nichts Abnormes zu constatieren. Ebensowenig am übrigen Körper. 
Nach diesem Befunde war es deutlich, daß wir einen Ductus omphaloentericus persistens vor uns hatten. Bei dem guten Allgemeinzustande des Kindes schien mir die einzig rationelle Therapie, nicht allein die abnorme Communication des Darmes mit der Außenwelt zu verschließen, sondem anch den abnormen zwischen Darm und Nabel sich befindenden Strang zu eliminieren und lierdurch den Patienten vor allen schädlichen Folgen, die solch ein an der Bauchwand adhärentes Divertikel des Darmes frülı oder spät haben kann, zu bewabren.

Oas Kind wurde also am 1. Oktober im Krankenhause anfgenommen und operiert.

Ich machte die Operation in rubiger Chloroformnarcose. Das prolabierte Divertikel, samt angrenzendem Granulom werden umschnitten und die Incision ungefähr $2 \mathrm{~cm}$ auf- und abwärts verlängert. Nach Eröff́nung des Peritoneums im oberen Teile der Wunde sicht man, daß von einer Dündarmschlinge ein Divertikel zum Nabel verläuft und dort in cinem Bruchsacke eintritt. An einzelnen Stellen bestehen Adhäsionen zwischen Bruchsack und Divertikel. Diese werden stumpf gelöst, und der Bruchsack an seiner Basis umschnitten, wonach man das Divertikel frei am Darme härgend vor sich hat. Es inseriert an der Convexität des Darmes und ist $3-4 \mathrm{~cm}$ lang. Es wird nun an seiner Hasis abgeklemmt und abgeschnitten. I) so entstandene Offnung wird durch eine Nahtreihe, die alle Schichten der Darmwand faßt, und darüber eine Lembertsche Nahtreihe gesehlossen. Diese Nähte werden quer zur Längsrichtung des Darmes angelegt. Hie Banchwunde wird in zwei Etagen verschlossen. Collodiumverband.

Den nächsten Tag hatte das Kind leichte Erscheinungen von gastroenteritis und abends eine Temperatur von 38,5\%. Ich verordnete cinige Dosen Calomel, wonach den folgenden Tag Temperatur und Verdaung normal waren und weiterhin auch blieben.

Am 7. Oktober wurden die Nähte fortgenommen. Die Wunde war per primam geheilt. Den 8. Oktober wurde das Kind entlassen. Es hatte während der ersten drei Tage post operationem $100 \mathrm{~g}$ an Gewicht abgenommen, wog jetzt aber wieder ebensoviel als am Tage der Operation.

Zwwei Monate später wurde das Kind auf Aufforderung nochmals gezeigt. Es sah voryüglich aus. Seine Verdaung war rollkommen normal. Die Narbe war kaum sichtbar. Gewicht 12 Pfund.

Wie bekannt, bildet der Ductus omphalo-entericus im frühen foetalen Leben die Verbindung zwischen Darmkanal und Dotterblase; und hat diese Verbindung sich in der 8 . bis 9 . Woche rückgebildet Das teilweise oder gänzliche Mestehenbleiben ist die Ursache von verschiedenen $\Lambda$ bnormitäten. Bleibt sie in ihrer ganzen Länge bestehen, dann steht also der Darm durch den Nabel in offener Communication mit der Außenwelt, und alle ర̈bergänge von einer wenig Schleim absondernden Fistel bis zu einem kompletten Anus praeternaturalis umbilicalis können die Folge sein.

Die Gefahr nun, die ein offener Ductus omphalo-entericus seinem Besitzer bereitet, hängt in erster Linie daron $a b$, ob der Darminhalt 
inanptsächlich längs des natürlichen Weges, odcr längs der Fistel wird herausbefördert. Im letzteren Falle wird die außer Fnnktion Stellung des ganzen dicken und eines Teiles des dünnen Darmes nicht ohne aehädliche Folgen bleiben; während bei Kindern, die sich nicht in sehr sorgfältiger P’lege befinden; bald ein hartnäckiges Eczem dor Bauchwand aultreten wird.

Yiel ernster ist aber eine zweite Complication, namentlich das Auftreten eines Darmprolapses. Beinalie alle Patienten zeigten schon als sic in ärztliche Behandlung kamen, einen Prolaps ron einem Teile des Divertikels selbst, der auch in meinem Falle bestand. Dieser Prolaps hat nun oft große Tendenz stets größer zu werden, bis dias ganze Iivertikel - bis zu seiner Insertion am Darme ausgestiulpt ist und dann bei noch weiterer Zunahme des Prolapses notwendigerweise anch der Darm selbst prolabieren muß. Die verschiedenen Stadien dieses Vorganges hat Barth (1) in einem deutlichen Schema abgebildet, das man in mehreren Fand- und Iehrbüchern finden kann. Die Incarceration dieses prolabierten Darmstückes ist nun eine weitere Complication, die öfters den Zustand noch in sehr ernster Weise verschlimmert. Bis heute ist nun, soviel ich dic Literatur übersehe, nur ein Fall bekannt, dab ein Patient mit offenem Ductus omphalo-entericus, wobei ein Darmprolaps zustande gekommen war, geheilt ist. Da dieser Fall, von Briddon (2) veröffentlicht, weder im Central.Blatt, noch in Hildebrandts Jabresbericbt referiert ist, erlaube ich mir, hier einen Auszug der Krankengeschichte mitzuteilen.

Der Patient war ein hnabe, der die ersten zehn Tage auf normalem Wege defaecierte. Fon da $a b$ fing der Darminhalt an, längs des offen gebliebenen Nabels auszutreten, und nach werigen Tagen hörte die Defaecation per anum ganz auf. Im Alter von 3 Wochen wurde das Kind in einem krankenhause aufgenommen. Es war schlecht genährt. $\Lambda$ n der Stelle des Nabels ragte ein mehr wie hïhnereigroler Darmprolaps hervor. Der dem zuführenden Schenkel entsprechende Teil des Prolapses hatte einen vier bis fünfmal größeren Lmfang wie der dem abführenden Schen$k$ el entsprechende Teil; an ersterem waren dentlich peristaltischo Bewegungen $z n$ sehen. Incarcerationserscheinnngen sehienen nicht za bestelien; es ist wenigstens dariiber nichts angegeben. Das Kind wurde narkotisiert und eine Ineision unmittelbar unterhalb des Nabels gemacht. Frst nachdem dio Öfnnng am Nabel dureh Schnitt erweitert war, golang es leicht, den Prolaps zil reponieren. Danach konnte man eonstatieren, dals es tatsächlich oin offenes Meckelsches Divertikel war, wodurch ein Teil des Ileum prolabiert gewesen war. Magrenwärts rom Divertikel waren die Darmschlingen stark erweitert; analwärts so stark verengt, dals man zweifelte, ob die Passage des Darminhalts cine ungestörte sein worde. Vorläufig wurde also die Öffnung des Divertikels als Anus praeternaturalis belassen. Die ersten 
zehn Tage post operationem wurde der ganze 1)arminhalt durch den Nabel heransbefördert. Dann wurde durch einen Tampon und Heftpflastercerband der Anus praeternaturalis abgeschilossen, was zur Folge batte, da.B die Defaecation ganz auf normalem Wege von statten ging. Als das Kind sieben Wochen alt war, wurde schließlich die Laparotomie und Resection des Divertikeis vorgenommen, welche Operation der Pationt gat ib berstand. Auch der Allgemeinzustand hatte sich in der Zeit sehr gebessert.

Wie gesagt, ist dies meines Wissens bisher der einzige mit Darmprolaps complicierte Fall, der geheilt ist; und dabei verkehrte der Patient, noch insoweit in günstigen Cinständen, daß trotz des groben Prolapses rioch keine Incareerationserscheinungen bestanden.

In keinem anderen Falle, wo es schon zum Darmprolaps und teilweise schon zur Incarceration des Prolapses gekommen war-solche Fälle sind von Barth (1), Hellweg (3), Weinlechner (4), Theremin (5), Arndt (6), Karewski (7) u. a. pabliciert - gelang es, den Pationten durchzubringen.

Endlich kann auch der abnorme zwischen Darm und Bauchwand verlaufende Strang in früher oder späterer Zeit sehr ernste Passagestörungen des Darmes verursachen. Derartige Fälle sind von Leis rink (8) und Walzberg (9) bekannt gegeben; aber auch hier vermochte die wegen des bestehenden Ileus unternommene Operation keine Rettung mehr zu bringen.

Der Gefahren, die einem Patienten mit offenem Ductus omphaloenterieus drohen, sind also viele; und es ist sehr begreiflich, daß auch für diese Krankbeit sich die Idee einer Radikaloperation aufdrängen mußte. Bartl war der erste, der 1887 diese Idee, wenn auch noch mit eirer gewissen Reserve, ventilierte, $\Lambda$ Isberg der erste, der sie 1888 zur Ausführung brachte. Diese Radikaloperation hat dann natürlich zu bestehen in der Laparotomie und Resection des ganzen Divertikels bis zu seiner Insertion am Darme. In den späteren Jahren wurde dieser Weg dann auch, beinahe immer mit gutem Resultate, schon des öfteren befolgt; und ieh glaube, daß man jetzt wohl als Regel aufstellen kann, daß bei einem an Persistenz des Ductus omphalo-entericus leidenden Kinde, das übrigens gesund ist, so bald wie möglich diese Radikaloperation vorgenommen werden soll.

Um einen Überblick über die bis jetzi auf diesem Wege erzielten Erfolge zu bekommen, habe ich in untenstehender Tabelle alle in der mir zugänglichen Titeratur publizierten Fälle zusammengestellt mit Angabe des Alters des Patienten und des Resultates der Operation

Operateur.

Alsberg (10)

Shepherd (11)

$\begin{array}{ccc}\text { Alter } & \text { Resultat } \\ 4 \text { Monate } & \text { exitus } \\ 3 \text { geheilt }\end{array}$




$\quad$ Operateur
Gevaert (12)
Petersen (13)
Herzog (14)
Fräkel (15)
Deschin (16)
Broca (17)
Broca
Broca
Stierlin (18)
Korte (19)
Karews k (7)
Morian (20)
Kraske (21)
Briddon (2)
Kirmisson (22)
Herbing (23)
Robinson (24)
Ifubbard (25)
Maas (26)
Sträter

\begin{tabular}{|c|c|}
\hline $\begin{array}{c}\text { Alter } \\
2^{1} / 2 \text {.Jahre }\end{array}$ & $\begin{array}{l}\text { Resultat } \\
\text { gebeilt }\end{array}$ \\
\hline $\bar{j} \quad y$ & \\
\hline 10 Monate & exitus \\
\hline 3 Wochen & zeheilt \\
\hline 5 Monate & exitus \\
\hline 6 & gebeilt \\
\hline 10 & $\because$ \\
\hline 6 & $"$ \\
\hline 2 & " \\
\hline 15 & , \\
\hline$?$ & $n$ \\
\hline s Wochen & $\%$ \\
\hline 1 Jahr & $y$ \\
\hline 7 Wochen & $\therefore$ \\
\hline 5 Monate & 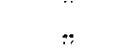 \\
\hline 6 Wochen & $"$ \\
\hline 13 Monate & $\because$ \\
\hline 4 Wochen & $n$ \\
\hline $8 \quad "$ & 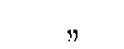 \\
\hline $4 \quad \%$ & " \\
\hline
\end{tabular}

Von diesen 22 Patienten sind also drei an den Folgen der Operation zugrunde gegangen. Auf den ersten Blick scheint dies für eine gewissermaßen prophylaktische Operation eine viel zu bohe Nortalitütsziffer. Wenn man sich aber die Krankengeschichten der ungünstig verlaufenen Fälle etwas näher ansicht, wird man eines besseren bolehrt.

Als ber g selbst schreibt den Mißerfolg seines Falles, der 12 Tage post operationem an Peritonitis zugrunde ging, auf Rechnung eines technischen Fehlers. Lir hatte einen kleinen Teil des Ductus zurückgelassen und dessen Lumen nur mit einer Sahtreihe, dic alle Schichten falte, geschlossen.

Auch der Patient von IIerzog starb, 3 Tage post operationem, an Peritonitis. In der Krankengeschichte ist angegeben, daß vor der Operation der Allgemeinzustand alles zu wünschen übrig läßt. Dabei bekommt das Kind noch am 7 . März eine Pneumonie und wird trotzdem am 18. März operiert.

Jer Patient fon Deschin fieberte vor der Operation. Während der Chloroformnarkose traten wiederholt Anfälle von Asphyxie auf. Gegen Ende der Operation standen Herz und Atmung still. Patient erholte sich etwas nach Injektion von Kampfer und pbysiologischer 
148 VIIT. Sträten, Zur Radikaloperat. d. Iuctus omphalo-entericus persistens.

Kochsalzlösung; starb aber $\mathbf{i}$ Stunden post operationem an zunehmender Werzschwäche.

In keiner Weise sprechen diese drei ungünstig verlaufenen Fälle also gegen die Richtigkeit der Behauptung, die ich als Schlußsatz meiner Erörtcrungen aufstellen möchte: Bei einem übrigens gesunden und kräftigen Kinde, das an Persistenz des Ductus omphalo-entericus leidet, ist sobald wie möglich die Laparotomie and Resection des Ductus vorzunehmen.

\section{Literaturverzeichnis.}

1) Barth, Deutsche Zeitschrift für Chir., Bd. 26.

2) Briddon, Annals of Surgery 1998.

3) Hellweg, Hospitals tidende 1884, ref. bei Barth.

4) Weinlechner, Jahrbuch für Kinderheilkunde, Bd. 8.

5) Theremen, Revue mensuelle des maladies de l'enfance 1855. Ref. bei Haie: Des fistules congenitales par persistance du conduit vitellin. Thèse de Paris 1897.

6) Arndt, Ref, bei Thebanlt: Des fistules congenitales par persistance du conduit vitellin. These de Paris 1898.

7) Karewski, Deutsche med. Wochenschr. 1898. Verciusbeilage Xr. 24.

8) Leisrink. Archiv für klin. Chir. Bd. $2 S$.

9) Walzberg, Verhandl. der Deutschen Ges. für Chir. 1595.

10) Alsberg, Deutsche med. Woehensehr. 1892, Nr. 46.

11) Shepherd, Arch. of Pediatries 1892 ref. Cbl. für Chir. 1892, Nr. 47.

12) Gevaert, Annales de med. et. de chir. 1892. Ref. Cbl. für Chir. 1894, Nir. 15.

13) Quact-Faslem, Das Offenbleiben des Ductus omphalo-mesentericus Inaug: Diss. Kiel 1899.

14) Sa uer, Deutsche Zeltschr. für Chirurgie, Bd. 44.

15) Neurath, Wiener klin. Wochenschr. 1896, Nr. 49.

16) Deschin, Chirurg. Annalen 1895. Ref. Cbl. für Chir. 189a, Nr. 50.

17) Bureau, Prolapsus ombilical du diverticale de Meckel. These de Paris 1898.

18) Stieriin, Deutsche med. Wochenschr. 189i, Ni. 12.

19) Körte, Deutsche med. Wochenschr. 1898 Nr. $t$.

20) Morian, Archiv für klin. Chir., Bd 58.

21) Kern, Beiträge zur klin. Chir., Bd. 19.

22) Kirmisson, Revue d'Orthopedie 1901, Xr. 5.

23) Herbing, Cbl. f. Chir. 1901, Nr. 48.

24) Robinson, Lancet 1902 , S. 302 .

25) Hobbard, Annals of Surgery, April 1902.

26) Maas, Berl. klin. Wochenschr. 1903, Nr. 25. 